\title{
The Role of Anticoagulation Clinics in the Era of New Oral Anticoagulants
}

\author{
Sophie Testa, Oriana Paoletti, Anke Zimmermann, Laura Bassi, \\ Silvia Zambelli, and Emilia Cancellieri
}

Haemostasis and Thrombosis Center, A.O. Istituti Ospitalieri, Cremona, Italy

Correspondence should be addressed to Sophie Testa, s.testa@ospedale.cremona.it

Received 24 August 2012; Accepted 13 September 2012

Academic Editor: Walter Ageno

Copyright (C) 2012 Sophie Testa et al. This is an open access article distributed under the Creative Commons Attribution License, which permits unrestricted use, distribution, and reproduction in any medium, provided the original work is properly cited.

\begin{abstract}
Anticoagulation Clinics (ACs) are services specialized in management of patients on anticoagulant treatment. At present, ACs manage patients chiefly on antivitamin $\mathrm{K}$ antagonists (AVKs), but patient population has already changed in the last few years, because of an increase of treatments with other anticoagulant drugs, which require different management systems. The strong increase in the number of patients at AC, mainly on long-term treatment, has determined the development of web management, through telemedicine systems, improving the quality of life and maintaining the same clinical quality levels. New oral anticoagulants (NOAs) have shown to be as effective as AVK antagonists in stroke prevention in atrial fibrillation and for treatment of venous thromboembolism in addition to VTE prophylaxis in orthopaedic surgery, when administered at a fixed dose, but patient adherence and compliance are crucial for good quality treatment. At present, lacking data from the real world, an oversimplification of treatment with NOAs could cause unjustified risks for patients and also a possible future underuse of good drugs. For these reasons the vigilance must be high and ACs can have a crucial role in defining which is the best management for NOA patients and how to do it, as it happened for AVKs.
\end{abstract}

\section{Introduction}

Anticoagulation Clinics (ACs) are services specialized in management of patients on anticoagulant treatment. They can be established as a section of a Thrombosis Centermultifunctional services with clinical and laboratory expertees, wich provide diagnosis, treatment and prevention of thromboembolic diseases- or act as independent services.

At present, ACs manage patients chiefly on antivitamin $\mathrm{K}$ antagonists (AVKs), but patient population has already changed in the last few years, because of an increase of treatments with other anticoagulant drugs, such as low molecular weight heparin (LMWH) or pentasaccharides, which require different management systems.

AVKs, that until few months ago were the only oral available anticoagulant agents, are life-saving therapies that can effectively prevent cardioembolic strokes related to atrial fibrillation and heart valve replacements and treat venous thromboembolism. Because of their characteristics AVKs need a strict laboratory and clinical control to ensure efficacy and safety [1]. In fact major bleeding and thrombotic complications are mainly related to coagulation levels out of ranges, age, comorbidities, comedications, and poor therapeutic control $[2,3]$. Therefore effectiveness and safety of AVKs increase when a good control of anticoagulation level is guaranteed. For this reason, a correct use of AVK requires a careful clinical and laboratory monitoring, as well as specific competences for managing complications and/or emergencies [1].

\section{Anticoagulation Clinic}

For AVKs we can consider 4 different management models: (1) routine medical care (RMC), (2) AC, (3) patient self testing (PST), and (4) patient self-management (PSM). The four models for AVK control are variously developed in different countries. Several studies have shown that anticoagulation management is crucial to ensure quality of treatment and, among different types of management, 
and Anticoagulation Clinic represent the best management model (Table 1) [4-13].

Aims of ACs are the following:

(a) determining the appropriate clinical indications for anticoagulant treatment;

(b) determining the laboratory tests necessary to pharmacological monitoring;

(c) prescribing the anticoagulation regimen based on the results of the laboratory tests;

(d) defining the time intervals for regular anticoagulation controls;

(e) assessing the potential pharmacological interactions;

(f) taking care of patients undergoing surgical interventions;

(g) carrying out educational programs for patients and healthcare providers.

The increase of AVK population in the last thirty years is related to many factors, including the good organization of ACs and the standardisation of laboratory methods, which has allowed to perform clinical trials in order to demonstrate AVK efficacy and safety [4]. These studies have supplied a better knowledge of therapeutic indications, optimal anticoagulation levels (therapeutic ranges), risk of haemorrhagic and thrombotic complications, pharmacological interferences, and the importance of a specialistic management [6].

\section{Anticoagulation Clinic and Telemedicine Development}

The strong increase in the number of patients at AC, mainly on long-term treatment, has determined the development of web management, through telemedicine systems $[14,15]$, in some areas.

Telemedicine systems for AVK have been developed during the last few years with the aim to decentralize in the health territorial care units the activity of ACs, improving the quality of life of patients living far from the AC site and maintaining the same clinical quality levels.

Until now, in daily clinical practice AVKs are still underused because they are often considered a therapy that can be hardly managed, laborious, and potentially dangerous for the patient, therefore an integration between hospital services and health care units can facilitate access to lifesaving therapies.

Telemedicine systems for anticoagulated patients should be structured through net supported programs to collect and elaborate clinical data, connected among hospital divisions, health care peripheral districts, and patients. All information, including clinical data, laboratory controls, alerts, prescriptions, and recommendations have to be available in real time through a bidirectional connection $[15,16]$.

Telemedicine applied to anticoagulated patients offers several advantages, both for organization as well as for improving communications. Decentralize clinical activity
TABle 1: Principal characteristics of the four management models.

\begin{tabular}{lcccc}
\hline & RMC & AC & PSM & PST \\
\hline Clinical quality & + & ++++ & ++++ & ++++ \\
TTR & + & +++ & +++ & ++++ \\
Accessibility & ++++ & ++ & + & ++ \\
Costs & + & ++ & +++ & ++++ \\
\hline
\end{tabular}

through a telemedicine system gives the opportunity to redistribute patient population into different health care areas, whose medical services could be otherwise underused. It also can help accessibility to health care services for an increased number of patients, empowering management possibilities. At the same time the web gives the opportunity to export quality procedures and competences outside of specialized centres, increasing communications, not only between the physician and patient, but also between different clinical specialties.

Telemedicine organizations are opportunities to improve health care and patient's quality of life through a capillary distribution of medical assistance, supporting physicians in the application of good clinical practice in anticoagulation management.

As anticoagulant drugs are changing through the introduction of new antithrombotic drugs, telemedicine could help their management.

New-generation antithrombotic drugs will need different types of management compared with AVKs, with a less frequent clinical and laboratory control. However, it will be necessary to follow up and record patient's data to guarantee the strict quality control on adherence, compliance, incidence of intercurrent diseases, adverse events, and complications.

The advantage of a web organization can be summarized as following:

(i) accessibility for a higher number of patients to a high quality management system;

(ii) integration among different specialists and health structures;

(iii) continuing medical record update;

(iv) possibility to manage patients on different antithrombotic drugs.

The web gives the possibility to increase quickly connections by modulating the management in relation to different molecules, assuring clinical control for all patients.

\section{New Oral Anticoagulant Drugs}

While AVK antagonists are indirect agents that act blocking liver synthesis of vitamin $\mathrm{K}$ coagulation factors, NOAs are synthetic molecules that inhibit directly a single target coagulation factor (Table 2) [17, 18]. New agents are already available in some countries: dabigatran, a direct anti-FIIa, and rivaroxaban, an anti-FXa. Apixaban and Edoxaban, two other anti-FXa, are in advanced evaluation state (see details in previous articles). 
Potential advantages as compared to VKA are:

(a) rapid onset of action without need for bridging therapy,

(b) predictable anticoagulant effect without need for dose-adjustment laboratory testing, and

(c) low food/drug interactions without need for restrictions.

Each agent has specific pharmacodynamic and pharmacokinetic characteristics (half-life, bioavailability, route of elimination, posology, frequency of administration, and potential drug interactions) that should be well known by prescribers (Table 2 ).

NOAs have shown to be as effective as AVK antagonists in stroke prevention in atrial fibrillation and for treatment of venous thromboembolism [19-22] in addition to VTE prophylaxis in orthopaedic surgery [23]. Studies published until now show similar safety, but a reduction in major cerebral bleeding for dabigatran, rivaroxaban, and apixaban [19-23].

Even if NOAs proved to be effective and safe when administered at a fixed dose, laboratory tests could be useful in clinical practice for measuring the anticoagulant effect in specific conditions such as: bleeding and thrombotic complications, surgery or invasive procedures, renal impairment, liver disease, and potential overdosages [24].

Several studies have been recently published showing different coagulation test sensitivity for each molecule [2427]. Currently few data are available to recommend the most appropriate test for each drug.

\section{Patient Management on NOA}

Patient adherence and compliance are crucial for NOA good quality treatment, but not for AVK patients, because the systematic laboratory controls improve the adherence to therapy. Usually, patients taking chronic drugs have poor medication-taking behavior [28]. Literature shows that even after an emergency, such as an acute coronary syndrome, the continuous use of life-saving drugs after 6-12 months drops to $71 \%$ for aspirin, $46 \%$ for beta-blockers, and $44 \%$ for statins, with the adherence to all the three medications being only $21 \%$ [29]. Estimates of nonadherence in the elderly (defined as those aged $\geq 65$ years) varies from $40 \%$ to $75 \%$, especially in chronic asymptomatic conditions like hypertension or as could be atrial fibrillation.

It would be desirable that clinical followup is organized. Among prescribing physician, general practitioner, other specialties, and Anticoagulation Clinic the last one has more competences, as previously described.

Alternatively, other less effective measures to improve compliance are:

(i) phone call by nurses,

(ii) $\log$ book to be filled by patients,

(iii) broad education at time of prescription, and

(iv) specific education with pamphlets and questionnaires.
At present, laboratory monitoring is not recommended in patients taking NOAs because phase III studies have shown efficacy and safety in administration at fixed dosage [19-23]. However particular clinical conditions in real world population will require and need laboratory control coagulation test to evaluate haemostatic balance.

For example:

(i) before surgery to assess haemostatic system and consequently the safety of invasive procedures,

(ii) in patients with bleeding/thromboembolic complications to evaluate a correlation between events and over-/undertreatment,

(iii) in elderly patients, who physiologically have renal impairment, or in underweight and obese patients to evaluate drug accumulation,

(iv) in renal/liver disease, and

(v) potential pharmacological interactions.

\section{The Anticoagulation Clinic in the Future}

If it is true that each type of anticoagulant drug has specific pharmacodynamic and pharmacokinetic characteristics, then we must consider different types of clinical/laboratory management in relation to their proper characteristics.

For all of them (AVK and NOA) we have to consider the following items (Table 3):

(1) determine the appropriate clinical indications for anticoagulant treatment;

(2) choose the best drug in relation to clinical patient profile;

(3) evaluate any potential pharmacological interference, in particular in the elderly;

(4) control drug adherence, that for AVKs is guaranteed through laboratory test;

(5) define a follow-up program;

(6) define educational programs to increase adherence;

(7) manage patients undergoing surgical interventions or invasive procedures;

(8) manage patients with bleeding and thrombotic complications, during anticoagulant treatment;

(9) manage patients during intercurrent diseases;

(10) choose the best laboratory test for each molecule to support clinical and therapeutical activities.

As previously discussed compliance of patients that require long-term therapy is a crucial problem and represents an important factor of variability of both quality of treatment and patient management. Lacking evidences for NOAs laboratory monitoring, it is required a strict control of compliance and adherence to avoid major complications due to under-/overdosages [30-35].

Educational courses for patients (indications for treatment, risk and benefit, problem in drug administration, 
TABLe 2: Principal characteristics of new oral anticoagulants ([18, 19], modified).

\begin{tabular}{lcccc}
\hline & Dabigatran & Rivaroxaban & Apixaban & Edoxaban \\
\hline Target & IIa & Xa & Xa & No \\
Prodrug & Yes & No & $1-3$ & No \\
Hours to $C_{\max }$ & 2 & $2-4$ & $66 \%$ & $50 \%$ \\
Bioavailability & $7 \%$ & $80 \%$ & $8-15$ & $6-11$ \\
Half-life (hours) & $12-14$ & $9-13$ & Yes & Yes \\
CYP metabolism & No & Yes & Yes & Yes \\
Efflux transporter P-gp & Yes & Yes & $25 \%$ & $35 \%$ \\
Renal elimination & $80 \%$ & Od (33\% cleared unchanged) & Bid \\
Dosing & Bid & & Od \\
\hline
\end{tabular}

TABle 3: Patients management: comparison between NOA and AVK clinical actions.

\begin{tabular}{lcc}
\hline & AVK & NOA \\
\hline Visit (anamnesis, physical examination) & Yes & Yes \\
Prescription (clinical indication, posology) & Yes & Yes \\
Information/education & Yes & Yes \\
Frequent lab coagulation monitoring & Yes & No \\
Renal function control & No & Yes \\
Dosing adjustment & Yes & No \\
Adherence/compliance control & No (routinely evaluated) \\
Management in special situations (surgery/complications) & Yes & Yes \\
Clinical periodical control & No (routinely evaluated) & Yes \\
Lab testing to support clinical and therapeutical intervention in specific condition & Yes & Yes (under evaluation) \\
\hline
\end{tabular}

dietary behaviour, interactions with other drugs, and intercurrent disease) and for health territorial care units can improve patient's compliance [36].

ACs are specialized services, well experienced on anticoagulant treatment and they are able to support patients on critical clinical conditions, mainly because of their knowledge of coagulation mechanisms. This means that they can easily understand complication etiopathogenesis and quickly provide specific therapeutic interventions in emergency conditions. As an example we can consider over dosages on AVK treatment and the correct use of prothrombin concentrates or preparation of patients on anticoagulant treatment for surgical intervention, through assessing the normalization of haemostatic parameters.

An advantage of ACs is also the complete recording of clinical and laboratory data, always available, necessary for clinical surveillance and pharmacovigilance evaluation.

Taking into account advantages provided by an $\mathrm{AC}$ an integration between hospital services and primary care units is desirable to standardize quality of treatment on anticoagulation. Telemedicine systems offer the opportunity to empower the integration among different heath care providers, ensuring standard level of care among specialists, capillary diffusion of anticoagulant treatments, homogeneous data collection, continuing education, real time evaluation of major complications and comparison among different drugs, and improving knowledge and information on NOAs.
This integration promotes communication among different professional activities (nurses, physicians) and it can help to control compliance and adherence of anticoagulated patients, assuring an optimal standard of care. In particular, in the initial period of the use of NOAs it is necessary a strict patient control to avoid errors in their clinical use, regarding correct indication, posology, adherence and compliance, patient management in special conditions like surgery, invasive procedures, or intercurrent diseases. At present, lacking data from the real world, an oversimplification of treatment with NOAs could cause unjustified risks for patients and also a possible future under use of good drugs.

For all these reasons, in these first years of introduction of NOAs, vigilance must be high and ACs can have a crucial role in defining which is the best management for NOA patients and how to do it, as it happened for AVKs.

\section{References}

[1] J. Ansell, J. Hirsh, E. Hylek, A. Jacobson, M. Crowther, and G. Palareti, "Pharmacology and management of the vitamin K antagonists: american College of Chest Physicians EvidenceBased Clinical Practice Guidelines (8th Edition)," Chest, vol. 133, no. 6, pp. 160S-198S, 2008.

[2] G. Palareti, N. Leali, S. Coccheri et al., "Bleeding complications of oral anticoagulant treatment: an inception-cohort, prospective collaborative study (ISCOAT)," Lancet, vol. 348, no. 9025, pp. 423-428, 1996. 
[3] G. Palareti, C. Manotti, A. D’Angelo et al., “Thrombotic events during oral anticoagulant treatment: Results of the inceptioncohort, prospective, collaborative ISCOAT study," Thrombosis and Haemostasis, vol. 78, no. 6, pp. 1438-1443, 1997.

[4] H. I. Bussey, R. M. Rospond, C. M. Quandt, and G. M. Clark, "The safety and effectiveness of long-term warfarin therapy in an anticoagulation clinic," Pharmacotherapy, vol. 9, no. 4, pp. 214-219, 1989.

[5] H. I. Bussey, E. Chiquette, and M. G. Amato, "Anticoagulation clinic care versus routine medical care: a review and interim report," Journal of Thrombosis and Thrombolysis, vol. 2, pp. 315-319, 1996.

[6] E. Chiquette, M. G. Amato, and H. I. Bussey, "Comparison of an anticoagulation clinic with usual medical care: Anticoagulation control, patient outcomes, and health care costs," Archives of Internal Medicine, vol. 158, no. 15, pp. 1641-1647, 1998.

[7] M. E. Cromheecke, M. Levi, L. P. Colly et al., "Oral anticoagulation self management and management by a specialist anticoagulation clinic: a randomised cross-over comparison," Lancet, vol. 356, no. 9224, pp. 97-102, 2000.

[8] J. Elston-Lafata, S. A. Martin, S. Kaatz, and R. E. Ward, "The cost-effectiveness of different management strategies for patients on chronic warfarin therapy," Journal of General Internal Medicine, vol. 15, no. 1, pp. 31-37, 2000.

[9] D. A. Fitzmaurice, C. Gardiner, S. Kitchen, I. Mackie, E. T. Murray, and S. J. Machin, "An evidence-based review and guidelines for patient self-testing and management of oral anticoagulation," British Journal of Haematology, vol. 131, no. 2, pp. 156-165, 2005.

[10] D. A. Fitzmaurice, E. T. Murray, D. McCahon et al., "Self management of oral anticoagulation: randomised trial," British Medical Journal, vol. 331, no. 7524, pp. 1057-1059, 2005.

[11] J. M. Garcia-Alamino, A. M. Ward, P. Alonso-Coello et al., "Self-monitoring and self-management of oral anticoagulation," Cochrane Database of Systematic Reviews, vol. 4, Article ID CD003839, 2010.

[12] D. B. Matchar, A. Jacobson, R. Dolor et al., "Effect of home testing of international normalized ratio on clinical events," New England Journal of Medicine, vol. 363, no. 17, pp. 16081620, 2010.

[13] C. Heneghan, A. Ward, R. Perera et al., "Self-monitoring of oral anticoagulation: systematic review and meta-analysis of individual patient data," Lancet, vol. 379, no. 9813, pp. 322334, 2012.

[14] C. Gardiner, K. Williams, I. J. Mackie, S. J. Machin, and H. Cohen, "Can oral anticoagulation be managed using telemedicine and patient self-testing? A pilot study," Clinical and Laboratory Haematology, vol. 28, no. 2, pp. 122-125, 2006.

[15] S. Testa, A. Alatri, O. Paoletti et al., "Reorganisation of an anticoagulation clinic using a telemedicine system: Description of the model and preliminary results," Internal and Emergency Medicine, vol. 1, no. 1, pp. 24-29, 2006.

[16] S. Testa, O. Paoletti, A. Zimmermann, L. Bassi, and E. Cancellieri, "Telemedicine for managing patients on oral anticoagulant therapy," in Telemedicine Techniques and Applications, vol. 15, pp. 331-346, 2011.

[17] J. W. Eikelboom and J. I. Weitz, "Update on antithrombotic therapy: new anticoagulants," Circulation, vol. 121, no. 13, pp. 1523-1532, 2010.

[18] B. I. Eriksson, D. J. Quinlan, and J. I. Weitz, "Comparative pharmacodynamics and pharmacokinetics of oral direct thrombin and factor Xa inhibitors in development," Clinical Pharmacokinetics, vol. 48, no. 1, pp. 1-22, 2009.
[19] S. J. Connolly, M. D. Ezekowitz, S. Yusuf et al., "Dabigatran versus warfarin in patients with atrial fibrillation," New England Journal of Medicine, vol. 361, no. 12, pp. 1139-1151, 2009.

[20] M. R. Patel, K. W. Mahaffey, J. Garg et al., "Rivaroxaban versus warfarin in nonvalvular atrial fibrillation," The New England Journal of Medicine, vol. 365, pp. 883-891, 2011.

[21] C. B. Granger, J. H. Alexander, J. J. McMurray et al., "Apixaban versus warfarin in patients with atrial fibrillation," The New England Journal of Medicine, vol. 365, pp. 981-992, 2011.

[22] C. Kearon, E. A. Akl, A. J. Comerota et al., "Antithrombotic therapy for VTE disease: antithrombotic therapy and prevention of thrombosis, 9th ed: American College of Chest Physicians evidence-based clinical practice guidelines," Chest, vol. 141, no. 2, supplement, pp. e419S-e494S, 2012.

[23] Y. Falck-Ytter, C. W. Francis, N. A. Johanson et al., "Prevention of VTE in orthopedic surgery patients. Antithrombotic therapy and prevention of thrombosis, 9th ed: American College of Chest Physicians evidence-based clinical practice guidelines," Chest, vol. 141, no. 2, supplement, pp. e278Se325S, 2012.

[24] V. Pengo, L. Crippa, A. Falanga et al., "Questions and answers on the use of dabigatran and perspectives on the use of other new oral anticoagulants in patients with atrial fibrillation. A consensus document of the Italian Federation of Thrombosis Centers (FCSA)," Thrombosis and Haemostasis, vol. 106, no. 5, pp. 868-876, 2011.

[25] D. D. Castellone and E. M. Van Cott, "Laboratory monitoring of new anticoagulants," American Journal of Hematology, vol. 85, no. 3, pp. 185-187, 2010.

[26] J. Douxfils, F. Mullier, S. Robert, C. Chatelain, B. Chatelain, and J. M. Dogné, "Impact of dabigatran on a large panel of routine or specific coagulation assays. Laboratory recommendations for monitoring of dabigatran etexilate2012," Thrombosis and Haemostasis, vol. 107, no. 5, pp. 985-997.

[27] M. M. Samama and C. Guinet, "Laboratory assessment of new anticoagulants," Clinical Chemistry and Laboratory Medicine, vol. 49, no. 5, pp. 761-772, 2011.

[28] M. Burnier, "Medication adherence and persistence as the cornerstone of effective antihypertensive therapy," American Journal of Hypertension, vol. 19, no. 11, pp. 1190-1196, 2006.

[29] L. K. Newby, N. M. Allen LaPointe, A. Y. Chen et al., "Longterm adherence to evidence-based secondary prevention therapies in coronary artery disease," Circulation, vol. 113, no. 2, pp. 203-212, 2006.

[30] M. K. Wychowski and P. A. Kouides, "Dabigatran-induced gastrointestinal bleeding in an elderly patient with moderate renal impairment," The Annals of Pharmacotherapy, vol. 46, no. 4, article e10, 2012.

[31] E. L. Cano and M. A. Miyares, "Clinical challenges in a patient with dabigatran-induced fatal hemorrhage," The American Journal of Geriatric Pharmacotherapy, vol. 10, no. 2, pp. 160163, 2012.

[32] E. Truumees, T. Gaudu, C. Dieterichs, M. Geck, and J. Stokes, "Epidural hematoma and intraoperative hemorrhage in a spine trauma patient on Pradaxa (dabigatran)," Spine, vol. 37, no. 14, pp. E863-E865, 2012.

[33] M. Legrand, J. Mateo, A. Aribaud et al., "The use of dabigatran in elderly patients," Archives of Internal Medicine, vol. 171, no. 14 , pp. 1285-1286, 2011.

[34] D. Fieland and M. Taylor, "Dabigatran use in a postoperative coronary artery bypass surgery patient with nonvalvular atrial fibrillation and heparin-PF4 antibodies," The Annals of Pharmacotherapy, vol. 46, article e3, no. 1, 2012. 
[35] M. Boland, M. Murphy, M. Murphy, and E. McDermott, "Acute-onset severe gastrointestinal tract hemorrhage in a postoperative patient taking rivaroxaban after total hip arthroplasty: a case report," Journal of Medical Case Reports, vol. 6, article 129, no. 1, 2012.

[36] D. Barcellona, P. Contu, and F. Marongiu, "Patient education and oral anticoagulant therapy," Haematologica, vol. 87, no. 10, pp. 1081-1086, 2002. 


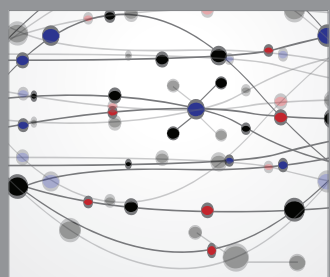

The Scientific World Journal
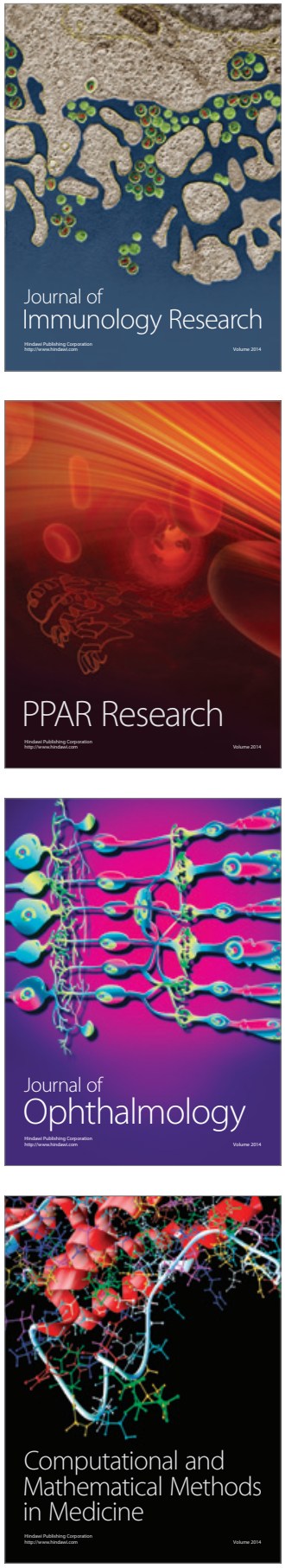

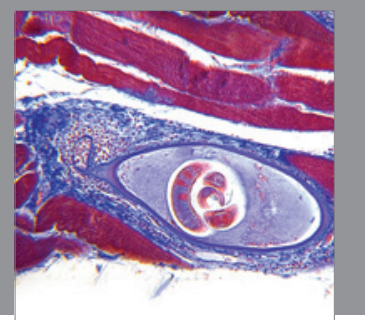

Gastroenterology

Research and Practice
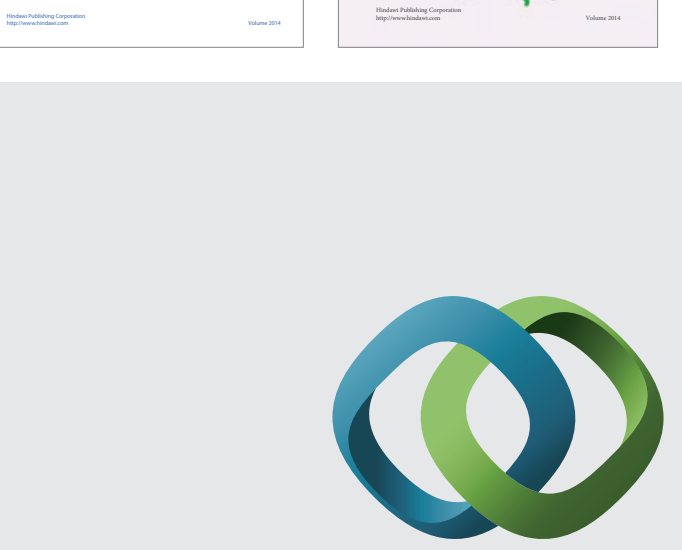

\section{Hindawi}

Submit your manuscripts at

http://www.hindawi.com
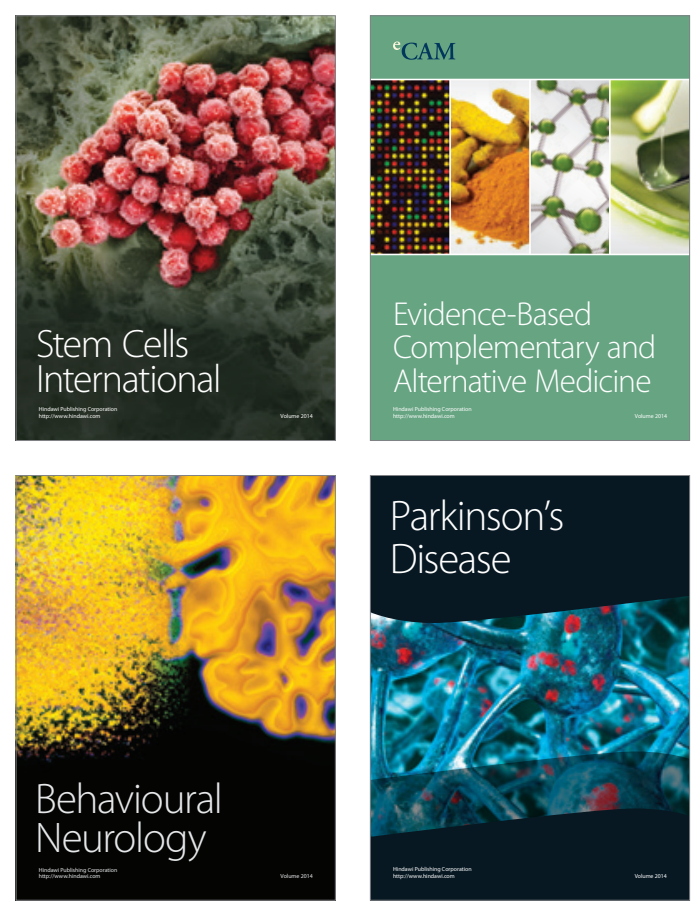

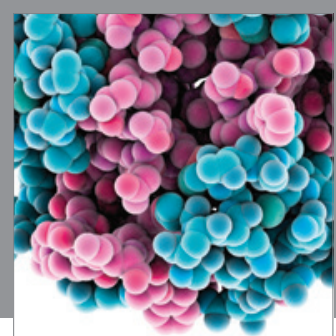

Journal of
Diabetes Research

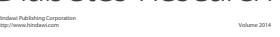

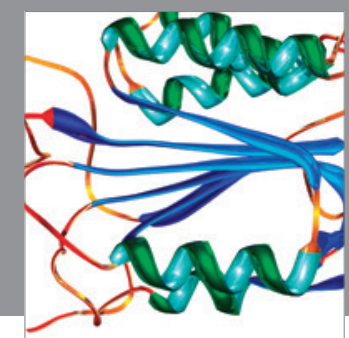

Disease Markers
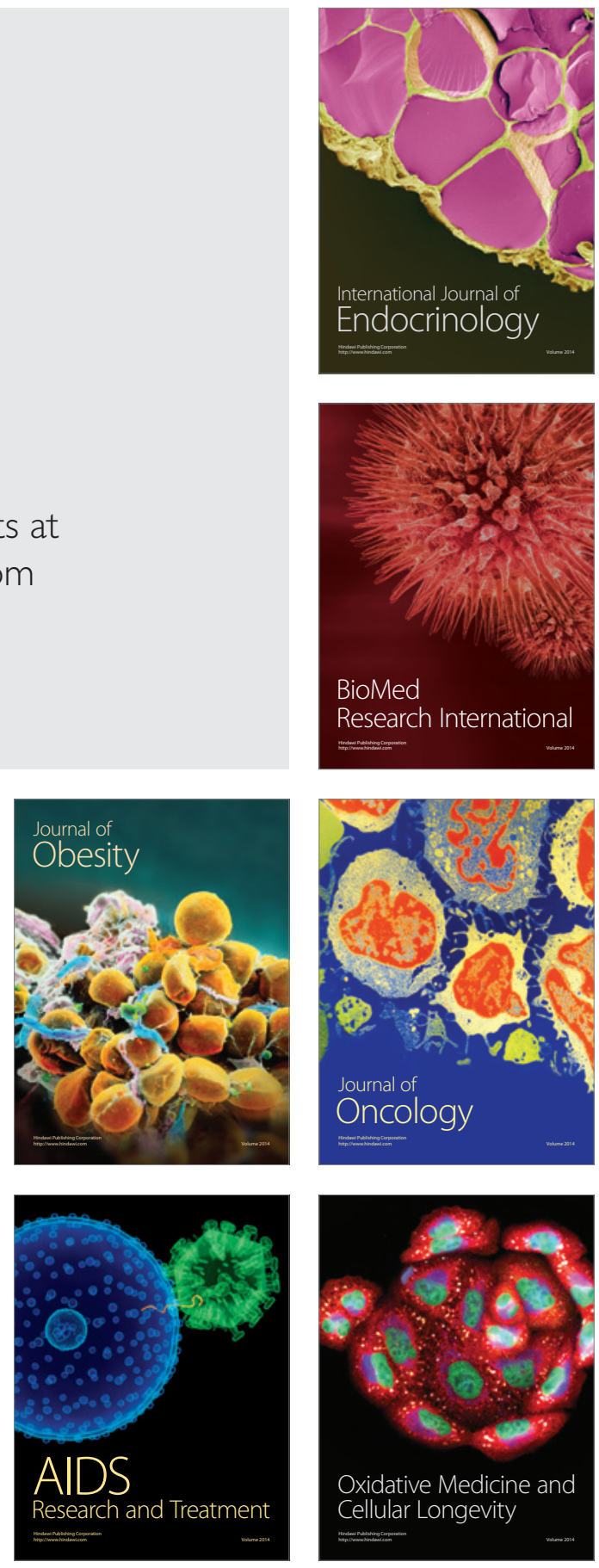